\title{
Influencing Factors and Driving Forces of Urbanization in Southeast Asia
}

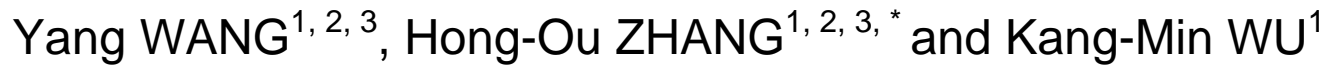 \\ ${ }^{1}$ Guangzhou Institute of Geography, Guangzhou 510070, China \\ ${ }^{2}$ Guangdong Open Laboratory of Geospatial Information Technology and Application, \\ Guangzhou 510070, China \\ ${ }^{3}$ Guangdong Academy of Innovation Development, Guangzhou 510070, China \\ *Corresponding author
}

\begin{abstract}
Keywords: Urbanization level, Population development, Grey relational degree, Southeast Asia, One Belt and One Road.
\end{abstract}

\begin{abstract}
In order to explore the driving factors to urbanization in Southeast Asia and correctly understand its functional mechanism and momentum, we analyzed the urbanization level and judged the urbanization development phase of different countries in Southeast Asia based on the perspective of population urbanization. Moreover, we explored the major driving force and influence strength of urbanization level on the basis of grey relational degree. The results show that: the urbanization level differences between various countries in Southeast Asia are distinct. Singapore, Brunei and Malaysia are countries of high urbanization level; Indonesia and the Philippines are countries of medium urbanization level; Cambodia, Burma, Laos, Vietnam and Thailand are countries of low urbanization level. Globalization, market environment and foreign capital are the core driving factors to the differences of urbanization level in Southeast Asian countries. Compared with previous studies, our research is more concerned about the impact of external factors on urbanization in Southeast Asia.
\end{abstract}

\section{Introduction}

Currently, the 'One Belt and One Road' strategy composed of 'Silk Road Economic Belt' and ' $21^{\text {st }}$ Century Maritime Silk Road' has entered into the all-round construction acceleration phase. The Southeast Asia region is adjacent to South China Sea, it's an inevitable passage and important cooperation platform for accelerating the Marine Silk Road Strategy from China, it's an important component in the relation pattern between China and the world, and it's also an important node area in the external economic route of China. The fast acceleration from urbanization is a distinguishing feature of the regional development in Southeast Asia in recent years. Therefore, the research on the regional geographical issues in Southeast Asia, particularly the urbanization issue is urgent and necessary.

From 1960 up to now, the various Southeast Asian countries have successively experienced very quick urbanization development period, the regional overall urbanization rate of Southeast Asia is increased from $22.7 \%$ in 1960 to $47.03 \%$ in 2014 [1]. Although it's still less than the world average level, the gap is gradually lessening. The population in Southeast Asian cities keeps increasing rapidly, it's increased from 157.53 million in 1990 to 29.4 million in 2014 [1], and it's nearly doubled over the 25 years, which has reflected the feature that the population urbanization is too fast [2]. This population is mainly gathered in the megacities surrounding the capitals [3]. It reflects the "megacity urbanization" phenomenon with proactive development of capital. Meanwhile, the urbanization quality in Southeast Asia is low, which is reflected on the disjunction of urban lifestyle conversion between growth of urban population and its employment nature, life quality and cultural and educational level, and is reflected with the "false urbanization" phenomenon. These features have posed many challenges toward the sustainable development from the cities in Southeast Asia [4]. The scholars have also noticed the huge differences between various cities in Southeast Asia in urbanization level and progress. The urbanization rate of Singapore has reached $100 \%$ very early, and the urbanization rate of Brunei and Malaysia also exceeds $70 \%$, all of them have entered the late 
period of urbanization, while the urbanization rate of Cambodia is only 20.38 (in 2013), and it's still in the initial stage of urbanization. Therefore, analyzing the new pattern of regional urbanization differences in Southeast Asia and clearing up the periodical features from different countries in urbanization development are significant to recognize the fundamental realities from various countries in Southeast Asia, to scientifically accelerate the construction of ' $21^{\text {st }}$ Century Maritime Silk Road' and China-ASEAN Free Trade Area.

Analysis on the driving factors to urbanization in Southeast Asia area is a basis to correct understanding of its functional mechanism and momentum. The existing research viewpoints are as follows: labor mobility, employment and population factors determine the urbanization of Southeast Asia [5]; the structural and institutional factors influence the urbanization process of Southeast Asia [6]; the regional development policies influence the pattern of urbanization in Southeast Asia [2]. As a whole, the research on driving factors to urbanization in Southeast Asia could be summarized as the factors including labor mobility, historical basis, economic level, regional policies, structural and institutional factors, national politics and social stability, etc. But with the influences from continuous deepening from the globalization tide on regional development, the influences from extraversion factors like globalization, market environment and foreign investment, etc on urbanization in Southeast Asia are becoming increasingly important. Based on this, this paper analyzes the major driving factors and influential strength to the differences in urbanization level of Southeast Asia area from four aspects including globalization degree, market environment, foreign investment drive and labor support on the basis of dual power perspective at home and abroad. This has an important academic significance on comprehensively understanding the urbanization features and fundamental realities from various countries in Southeast Asia, and it has important practical significance and reference value on scientifically accelerating the 'One Belt and One Road' strategy and China-ASEAN Free Trade Area construction and customizing the differentiated regional cooperation route between China and the Southeast Asian countries.

\section{Data and Methods}

\section{Research Area and Data Source}

We take 10 countries in Southeast Asia including Brunei, Cambodia, Indonesia, Laos, Malaysia, Burma, Philippines, Singapore, Thailand and Vietnam as research area, excluding East Timor. The total population of this area is 616 million, and the urban population is 283 million. The data collection year is 2013. The data mainly comes from 'The International Statistical Yearbook-2014', the 'national data' from the State Statistics Bureau and the 'ASEAN Statistical Yearbook 2013' [7].

\section{Judge the Major Driving Factors of Urbanization Level by Using Gray Relational Degree}

The relations between many factors in the geosystem are gray, it's difficult to distinguish the dominant factors and non-dominant factors and the relations between various factors. The gray correlation analysis provides a feasible approach for solving this kind of problems. As for the factors between 2 systems, the measurement of its relevance with changes of time or different objects is called as correlation. The correlation obtained by using gray correlation analysis is the "gray correlation”. The calculation step is listed as follows [8]:

The extremum method is employed for data standardization, the standardized value of the $k^{\text {th }}$ country in a certain factor sequence is:

$$
x_{k}=\left(F_{k}-F_{\min }\right) /\left(F_{\max }-F_{\min }\right)
$$

The gray relational coefficient of the $k^{\text {th }}$ country factors $i$ and $j$ is: 


$$
\xi_{i j}(k)=\frac{\min _{i} \min _{j}\left|x_{i}(k)-x_{j}(k)\right|+\rho \max _{i} \max _{j}\left|x_{i}(k)-x_{j}(k)\right|}{\left|x_{i}(k)-x_{j}(k)\right|+\rho \max _{i} \max _{j}\left|x_{i}(k)-x_{j}(k)\right|}
$$

The gray relational degree of factor $i$ and $j$ is:

$$
R_{i j}=\frac{1}{m} \sum_{k=1}^{m} \xi_{i j}(k)
$$

In the formula, $F_{k}$ is the original value of the $k^{\text {th }}$ country in a certain factor sequence; $F_{\max }$ and $F_{\min }$ are respectively the maximal value and minimal value of this sequence; $x_{k}$ is the numerical value of the $k^{\text {th }}$ country after standardization; $x_{i}(k)$ and $x_{j}(k)$ are respectively the standardized values of the factors from the $k^{\text {th }}$ country- $i$ and $j ; \xi_{i j}(k)$ is a correlation coefficient of factor $x_{j}$ and $x_{i}$ in the $k^{\text {th }}$ country; $R_{i j}$ is the gray degree of association between factor $I$ and factor $j$; $\rho$ is a resolution ratio, the general value is $0.5 ; m$ is the country number. In order to facilitate the quantitative analysis and to use the predecessor's research experiences for reference [8], the gray relation degree is classified into 5 grades according to different intensity indices: $0 \sim 0.2$ is a very weak correlation, $0.2 \sim 0.4$ is a weak correlation, $0.4 \sim 0.6$ is a medium correlation, $0.6 \sim 0.8$ is a strong correlation, and $0.8 \sim 1.0$ is a very strong correlation. This paper defines the strong correlation as a driving factor with distinct influences on urbanization.

\section{Results and Analyses}

\section{The Differentiated Features and Formation Causes to Population Urbanization Level in Southeast Asia}

It's classified into three classes according to the urbanization level of Southeast Asian countries, respectively high urbanization level countries, medium urbanization level countries and low urbanization level countries (Fig. 1).

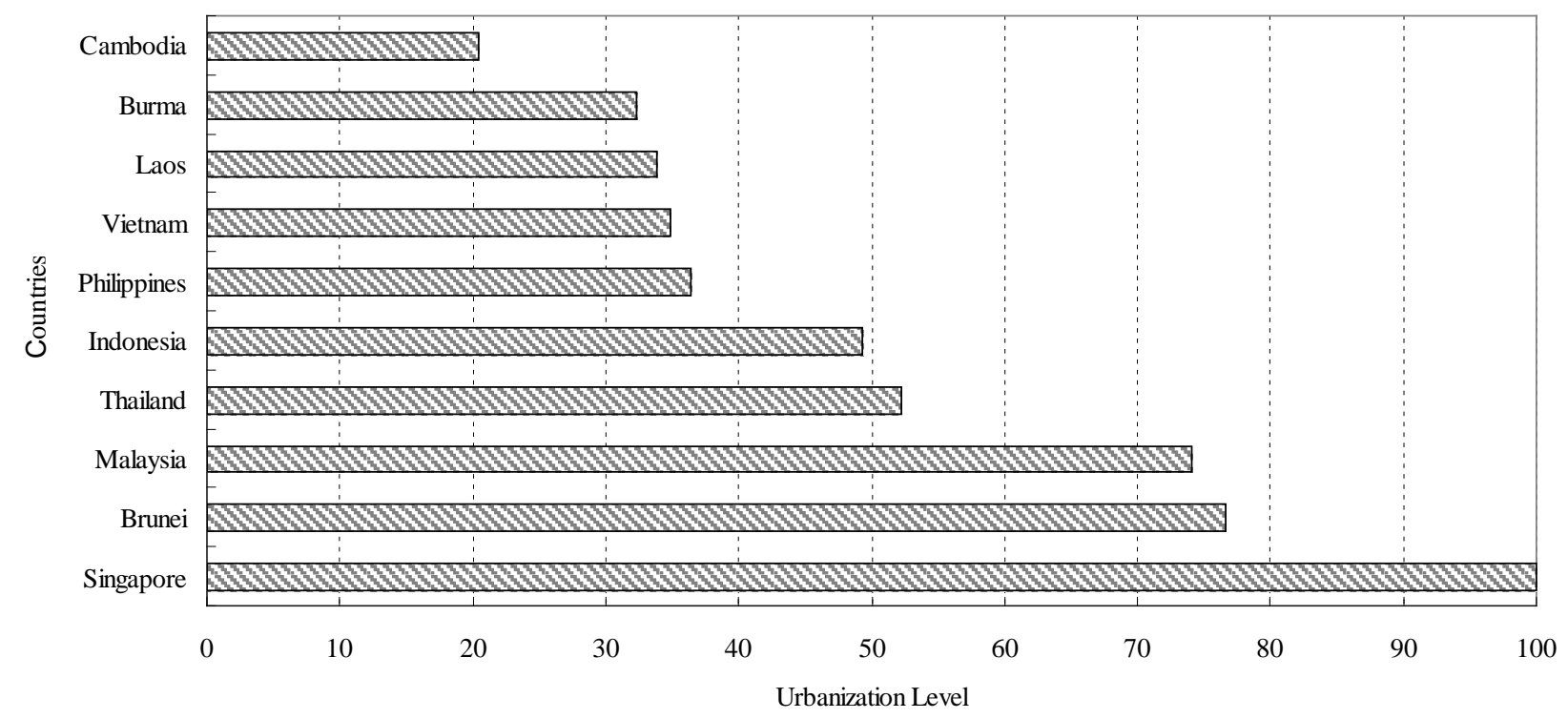

Figure 1. Difference characteristics of urbanization rate in Southeast Asia

The first, high urbanization level countries include Singapore, Brunei and Malaysia, which are in the late period of urbanization development, and urbanization is tending to be stable and mature. Among them, Singapore has started the industrialization process in 1960s, they belong to foreign trade driving economy, with a very high globalization degree, with business prosperity, and they are 
one of the global financial centers and traffic centers, and their development level is still in a top level in the world. Brunei is rich in petroleum and natural gas resources, the oil and gas exploitation started in 1970s and the development of public service industry have accelerated the non-agriculturization of the industry and have accelerated the urbanization process. The export-oriented economy is carried out in Malaysia since 1970s, its economy is rapidly developed since 1987, and it becomes a diversified newly-increasing industrial country and newly-rising market economy. Meanwhile, the population is rapidly gathering toward the cities, and the population urbanization speed even exceeds the economic urbanization speed, and they are in the late urbanization period with proactive population urbanization.

The second, medium urbanization level countries include the Philippines and Indonesia, which are in the accelerated development period of urbanization, with the urbanization rate about $50 \%$. These two countries transform their economic structures after the world war, with accelerated industrialization process. Meanwhile, the focus from government on development is in cities, as a result, the countryside is further lack of chances for development, hence the countryside is continuously fell behind and poor, and more and more labors are coming to cities from countryside to seek for the way out. Therefore, the labors are coming to cities mainly due to attraction from cities not due to push from countryside. Thus, the growth of urban population and the true urbanization (employment nature, life quality and cultural educational level, etc are all transferred into urban lifestyle) are seriously disjointed.

The third, low urbanization level countries include five countries-Laos, Thailand, Burma, Vietnam and Cambodia. The population urbanization level is low. Among them, the population urbanization level of Thailand, Burma, Vietnam and Cambodia is $30 \%-40 \%$, is striding forward toward the accelerated development period, while the urbanization level of Cambodia is still lingering at the initial stage, and is below 30\%. Because these countries are economically backward, with a very low overall development level, in addition the countries as Laos, Vietnam and Burma, etc have fell into wars and political unrests for a long time, they have no time in economic production and urban construction, as a result, their urbanization is in a low level. While in Thailand, the urban areas are very developed in modern economy, the countryside areas have backward agricultural natural economy, so the dual society with urban-rural division is established. But different from Indonesia and the Philippines, there is still a big population (most of them are poor people) living in countryside during the industrialization of Thailand.

\section{Driving Force to the Differentiated Features of Urbanization Level in Southeast Asia Area}

The driving forces of urbanization are always diversified [9], existing researches indicated that the driving factors to the urbanization in Southeast Asia are driven by labor mobility [6] and foreign investments etc, while these 2 factors are also related to the globalization degree and market environment of these countries. Therefore, this paper classified the driving factors to urbanization in Southeast Asia area as globalization degree, market environment, foreign investment and labor support. Among them, the globalization tide is an important motivating factor to the current urbanization, and it's indicated with the globalization index calculated by KOF Swiss Economic Societies. Market environment is an important fundamental factor for attracting enterprises to settle and to accelerate industry development, it's one of the engines of urbanization, and it's indicated with the index the percentage of enterprising opening costs in the per capita income. The lower is the value the better is the market environment or investment and venture environment. Therefore, this independent variable is converted with reciprocal. Foreign investment is an important factor of driving local economic development, creates employment opportunities and attracts agricultural population to flow toward cities, the per capita foreign investors' direct investment amounts are used to show this. Labor support is a fundamental factor in urbanization process, is the direct outward manifestation of 'urban pull' and 'rural push', which is shown with the percentage of labor population in the entire population. Among the aforementioned factors, globalization and foreign investments belong to external power, and labor support is internal power, the market environment has dual attributes of internal power and external power. As shown in Table 1, the globalization 
degree of Singapore is the highest, Malaysia is second to it, and Laos is the lowest. As for market environment, the enterprise opening costs of Burma and Cambodia accounts for $176.70 \%$ and $150.60 \%$ in per capita incomes, their enterprise opening costs are very high, with a bad market environment, while Singapore is only $0.60 \%$, with a good market environment; as for foreign investment driving, the per capita direct investment amount from foreign investors is 10492.48 USD in Singapore, with active foreign investments, but the one in the Philippines is only 28.43 USD, the one in Laos is 43.43 USD, and the one in Burma is 42.11 USD, so their foreign investments are also low; as for labor support, the percentage of labor population from the Philippines and Malaysia is low in the entire population, the one of the Philippines is $42.94 \%$ and the one of Malaysia is $43.89 \%$.

Table 1. Differentiated features of urbanization driving factors in various countries of Southeast Asia

\begin{tabular}{|l|l|l|l|l|}
\hline $\begin{array}{l}\text { Driving } \\
\text { factors }\end{array}$ & Globalization & Market environment & Foreign investment driving & Labor support \\
\hline $\begin{array}{l}\text { Evaluation } \\
\text { index }\end{array}$ & $\begin{array}{l}\text { Globalization } \\
\text { indicators }\end{array}$ & $\begin{array}{l}\text { Percentage of enterprise opening } \\
\text { costs in per capita incomes (\%) }\end{array}$ & $\begin{array}{l}\text { Per capita foreign } \\
\text { investors' direct } \\
\text { investment amount } \\
\text { (USD/person })^{-1}\end{array}$ & $\begin{array}{l}\text { Percentage of } \\
\text { labor population } \\
(\%)\end{array}$ \\
\hline Brunei & 65.69 & 9.90 & 2034.54 & 47.80 \\
\hline Cambodia & 49.04 & 150.60 & 102.87 & 56.86 \\
\hline Indonesia & 56.41 & 20.50 & 79.45 & 48.14 \\
\hline Laos & 27.07 & 6.70 & 43.43 & 50.36 \\
\hline Malaysia & 79.55 & 7.60 & 339 & 43.89 \\
\hline Burma & 32.06 & 176.70 & 42.11 & 59.02 \\
\hline Philippines & 57.40 & 18.70 & 28.43 & 42.94 \\
\hline Singapore & 88.63 & 0.60 & 10492.48 & 56.91 \\
\hline Thailand & 65.34 & 6.70 & 128.44 & 59.18 \\
\hline Vietnam & 48.58 & 7.70 & 93.28 & 59.91 \\
\hline
\end{tabular}

Based on this, gray relational analysis is performed on the urbanization level of Southeast Asia Area and the 4 aforementioned factors to calculate the gray relational degree (Table 2). The results indicate the gray relational degree between 3 factors (foreign investment drive, market environment and globalization degree) and urbanization level are both above 0.8, which is a high relevance. It indicates the 3 aforementioned factors are core factors of driving urbanization of the Southeast Asian countries, the gray relational degree of foreign investment drive and urbanization level is 0.894 432, with the most distinct driving effect. The labor support and urbanization level belong to medium relational degree, which indicates although labor support has a driving effect on urbanization of the Southeast Asian countries, the functioning degree is not distinct. This indicates the influences from external power with foreign investments and globalization as representative on the urbanization of Southeast Asian countries are stronger than the internal power with domestic labor support as representative, while market environment is also an important fundamental factor in attracting foreign investments, and intensifies the external drive of urbanization. These external powers accelerate the industrialization process of the Southeast Asian countries, therefore, promote the population to gather toward cities, and accelerate the urbanization process. Therefore, the differences of urbanization level between Southeast Asian countries are mainly driven by external factors. 
Table 2. Grey correlation degree between the urbanization levels its 4 driving factors in Southeast Asia

\begin{tabular}{|l|l|}
\hline Driving factors & Grey correlation degree \\
\hline Globalization & 0.824736 \\
\hline Market environment & 0.857504 \\
\hline Foreign investment driving & 0.894432 \\
\hline Labor support & 0.558095 \\
\hline
\end{tabular}

\section{Conclusions}

The differences in urbanization level between various Southeast Asian countries are distinct. The urbanization levels of Singapore, Brunei and Malaysia are very high, and they are in the advanced stage of urbanization development; the urbanization levels of the Philippines and Indonesia are medium; while the urbanization levels of Cambodia, Thailand, Burma, Laos and Vietnam are low, and they are in the initial stage.

The globalization, market environment and foreign investment drive are core driving factors to the urbanization level differences of Southeast Asian countries, the labor support factors are also influential, but the driving strength is very weak, and it's reflected the influences from external factors of a country on urbanization are stronger than the internal factors.

Comparing with the previous researches on driving factors to the urbanization in Southeast Asia, this research highlights on external factor influences from globalization and foreign investments, focuses on the domestic-overseas dual driving forces of urbanization, and provides new perspectives and references to relevant researches.

\section{Acknowledgement}

This research was financially supported by the National Natural Science Foundation of China (41401164), the Technology Plan of Guangzhou (201609020002), the Scientific Platform and Innovation Capability Construction Program of GDAS (2016GDASPT-0210), and the High-level Leading Talent Introduction Program of GDAS (2016GDASRC-0101).

\section{References}

[1] United Nations, Department of Economic and Social Affairs, Population Division, World Urbanization Prospects: The 2014 Revision, Highlights (ST/ESA/SER.A/352), United Nations Department of Economic \& Social Affairs Population Division, New York, 2014.

[2] G. W. Jones, Urbanization trends in Southeast Asia: some issues for policy, Journal of Southeast Asian Studies. 19 (1988) 137-154.

[3] G. W. Jones, Southeast Asian Urbanization and the Growth of Mega-Urban Regions, Journal of Population Research. 19 (2002) 119-136.

[4] K. S. Yap, T. Moe, Urbanization in Southeast Asia: Issues and impacts, Institute of Southeast Asian Studies, Singapore, 2012.

[5] T.G. McGee, Labor markets urban systems and the urbanization process in Southeast Asian countries, Population Institute, Honolulu, Hawaii, East-West Center, 1982.

[6] M. Liu, Y. Yin, Human Development in East and Southeast Asian Economies:1990-2010, Human Development Research Papers.17(2010) 1-328.

[7] A Secretariat, ASEAN Statistical Yearbook 2013,ASEAN Secretariat, Jakarta, 2014. 
[8] Y. Wang, D. L. Wang, L. H. Liu, et al, Spatial Differentiation of Urban Housing Prices and Its Impacts on Land Market in China, China Land Science. 29 (2015) 33-40. (In Chinese)

[9] X. Q. Lin, Y. Wang, S. J. Wang, et al, Spatial differences and driving forces of land urbanization in China, Journal of Geographical Sciences. 25 (2015) 545-558. 